\title{
Candidate counterparts to the soft gamma-ray flare in the direction of LS I +61303
}

\author{
A. J. Muñoz-Arjonilla ${ }^{1,2}$, J. Martít, ${ }^{1,2}$ J. A. Combi ${ }^{1,5}$, P. Luque-Escamilla ${ }^{3,2}$, J. R. Sánchez-Sutil ${ }^{2}$, \\ V. Zabalza ${ }^{4}$, and J. M. Paredes ${ }^{4}$
}

1 Departamento de Física, EPS, Universidad de Jaén, Campus Las Lagunillas s/n, Edif. A3, 23071 Jaén, Spain e-mail: [ajmunoz; jmarti; jcombi]@ujaen.es

2 Grupo de Investigación FQM-322, Universidad de Jaén, Campus Las Lagunillas s/n, Edif. A3, 23071 Jaén, Spain e-mail: jrssutil@ujaen.es

3 Dpto. de Ing. Mecánica y Minera, EPS, Universidad de Jaén, Campus Las Lagunillas s/n, Edif. A3, 23071 Jaén, Spain e-mail: peter@ujaen.es

4 Departament d'Astronomia i Meteorologia and Institut de Ciències del Cosmos (ICC), Universitat de Barcelona (UB/IEEC), Martí i Franquès 1, 08028 Barcelona, Spain e-mail: vzabalza@am.ub.es; jmparedes@ub.edu

5 Fac. de Ciencias Astronómicas y Geofísicas, Universidad Nacional de la Plata, Paseo del Bosque, B1900FWA La Plata, Argentina

Received 24 November 2008 / Accepted 21 January 2009

\section{ABSTRACT}

\begin{abstract}
Context. A short duration burst reminiscent of a soft gamma-ray repeater/anomalous X-ray pulsar behaviour was detected in the direction of LS I +61303 by the Swift satellite. While the association with this well known gamma-ray binary is likely, a different origin cannot be excluded.

Aims. We explore the error box of this unexpected flaring event and establish the radio, near-infrared and X-ray sources in our search for any peculiar alternative counterpart.

Methods. We carried out a combined analysis of archive Very Large Array radio data of LS I +61 303 sensitive to both compact and extended emission. We also reanalysed previous near infrared observations with the $3.5 \mathrm{~m}$ telescope of the Centro Astronómico Hispano Alemán and X-ray observations with the Chandra satellite.

Results. Our deep radio maps of the LS I +61 303 environment represent a significant advancement on previous work and 16 compact radio sources in the LS I +61 303 vicinity are detected. For some detections, we also identify near infrared and X-ray counterparts. Extended emission features in the field are also detected and confirmed. The possible connection of some of these sources with the observed flaring event is considered. Based on these data, we are unable to claim a clear association between the Swift-BAT flare and any of the sources reported here. However, this study represents the most sophisticated attempt to determine possible alternative counterparts other than LS I +61 303.
\end{abstract}

Key words. X-ray: stars - radio continuum: stars - infrared: general - X-rays: binaries - gamma rays: observations

\section{Introduction}

LS I +61 303 (V615 Cas) is a gamma-ray binary originally discovered in 1977 in the radio during a survey for variable sources in the Galactic plane (Gregory \& Taylor 1978; Gregory et al. 1979). The orbital period is about $26.5 \mathrm{~d}$ (Hutchings \& Crampton 1981) and it has been detected in data of frequencies between radio (Taylor \& Gregory 1982, 1984) and high energy gamma-rays (Albert et al. 2008). The physical interpretation of the LS I +61 303 emission across the complete electromagnetic spectrum still remains a matter of debate (Romero et al. 2007). Two different models have been proposed to explain the full spectral energy distribution: (i) a microquasar X-ray binary (Bosch-Ramon et al. 2006), and (ii) a nonaccreting pulsar interacting with the envelope of the rapidly rotating Be star (Dubus 2006).

On 2008 September 10, the Swift Burst Alert Telescope (BAT) detected a burst in the direction of LS I +61 303 within its 15-150 keV energy range (De Pasquale et al. 2008). The calculated location of this soft gamma-ray short-duration burst-event was found to have an error of 2.2 and the position of LS I +61 303 was found to be clearly consistent with this event (Barthelmy et al. 2008). Given this coincidence, this burst was associated with magnetar-like activity linked to a young highly magnetized pulsar in the binary system (Dubus \& Giebels 2008). Unusual X-ray activity (hard high flux and QPOs) had also been reported by Ray et al. (2008) just a few weeks before based on PCA data from the RXTE satellite. If the QPOs originate in LS I +61 303, an accretion disk would be necessary to explain the nature of this source. However, the PCA field of view of $\sim 1$ degree includes many additional sources that could be responsible for the QPO behaviour.

Despite these facts, one cannot exclude the possibility of another unrelated source being responsible for the observed gamma-ray flare. A population of faint X-ray sources in the vicinity of LS I +61 303 were reported by Rea \& Torres (2008), who suggested that one of these sources might be the quiescent counterpart of a new transient magnetar. Muñoz-Arjonilla et al. (2008) also reported additional X-ray 
Table 1. Radio, near infrared, and X-ray observations of LS I +61 303 field used in this paper.

\begin{tabular}{lllcr}
\hline \hline & Instrument & \multicolumn{1}{c}{ Date } & Band & Integ. time \\
\hline \multirow{2}{*}{ Radio } & \multirow{2}{*}{ VLA CnD } & 08-Jun.-1992 & $\mathrm{C}(6 \mathrm{~cm})$ & $9.8 \mathrm{~h}$ \\
& & 27-Jun.-1992 & $\mathrm{L}(20 \mathrm{~cm})$ & $3.0 \mathrm{~h}$ \\
& & 09-Sep.-1993* & $\mathrm{C}(6 \mathrm{~cm})$ & $7.8 \mathrm{~h}$ \\
& & 13-Sep.-1993* & $\mathrm{C}(6 \mathrm{~cm})$ & $7.9 \mathrm{~h}$ \\
\hline \multirow{3}{*}{ Infrared } & \multirow{2}{*}{ CAHA 3.5 m } & 25-Sep.-2007 & $J(1.25 \mu \mathrm{m})$ & $905 \mathrm{~s}$ \\
& & 25-Sep.-2007 & $H(1.65 \mu \mathrm{m})$ & $905 \mathrm{~s}$ \\
& & 25-Sep.-2007 & $K_{\mathrm{s}}(2.18 \mu \mathrm{m})$ & $1811 \mathrm{~s}$ \\
\hline X-rays & \multirow{2}{*}{ Chandra } & 08-Apr.-2006 & 0.5-7.0 keV & $49.9 \mathrm{ks}$ \\
\hline
\end{tabular}

* Already used by M98.

and radio sources coincident with the Swift-BAT error circle and some of them with stellar-like counterparts.

We present extensive radio, X-ray, and near infrared observations of the Swift-BAT error circle at the location of this 2008 September 10 event. Both archival data and observations conducted by the authors were used in this paper as described in the $\log$ in Table 1. Different populations of sources were detected and their main observational properties are reported. A few interesting objects in the direction of the magnetar-like flare are highlighted and the possibility of them being alternative candidate counterparts is assessed. The census of radio/X-ray sources reported here represents the most complete study to date of alternative counterpart candidates to the Swift-BAT event.

\section{Radio sources within the Swift-BAT error circle}

The magnetar-like event that renewed interest in studying LS I +61 303 is probably related to a compact object formed as a result of a supernova event. Therefore, both compact and extended radio features could play a role in our understanding of this phenomenon. The environment of LS I +61 303 in the radio was studied by Martí et al. (1998, hereafter M98) at $6 \mathrm{~cm}$ wavelength using the very large array (VLA) of the national radio astronomy observatory (NRAO) with the array in its $\mathrm{CnD}$ configuration providing appropriate sensitivity to both compact and extended sources. We developed the M98 approach improving their sensitivity by combining additional $6 \mathrm{~cm}$ VLA archive data acquired by the same compact array configuration (see Table 1) to a total of $25.5 \mathrm{~h}$ of on-source time.

The AIPS package of NRAO was used for the standard interferometer data processing and self-calibration. We removed the variable LS I +61 303 core to avoid artifacts and replaced it for cosmetic reasons with a constant point source component with the observed average flux density. We also removed a nearby bright radio source, whose presence affected significantly the dynamic range of the maps. The final result is presented in Fig. 1, where extended emission is enhanced with a slight taper. Compact sources are more clearly evident in the non-tapered radio map of Fig. 2 with a rms noise of $13 \mu \mathrm{Jy}$ beam $^{-1}$, significantly lower than that of M98. The observational properties of these compact radio sources are listed in Table 2, where J2000.0 positions are given.

\section{Near infrared observations of the Swift-BAT error circle}

The field of LS I +61 303 was observed at near infrared wavelengths with the $3.5 \mathrm{~m}$ telescope at the Centro Astronómico

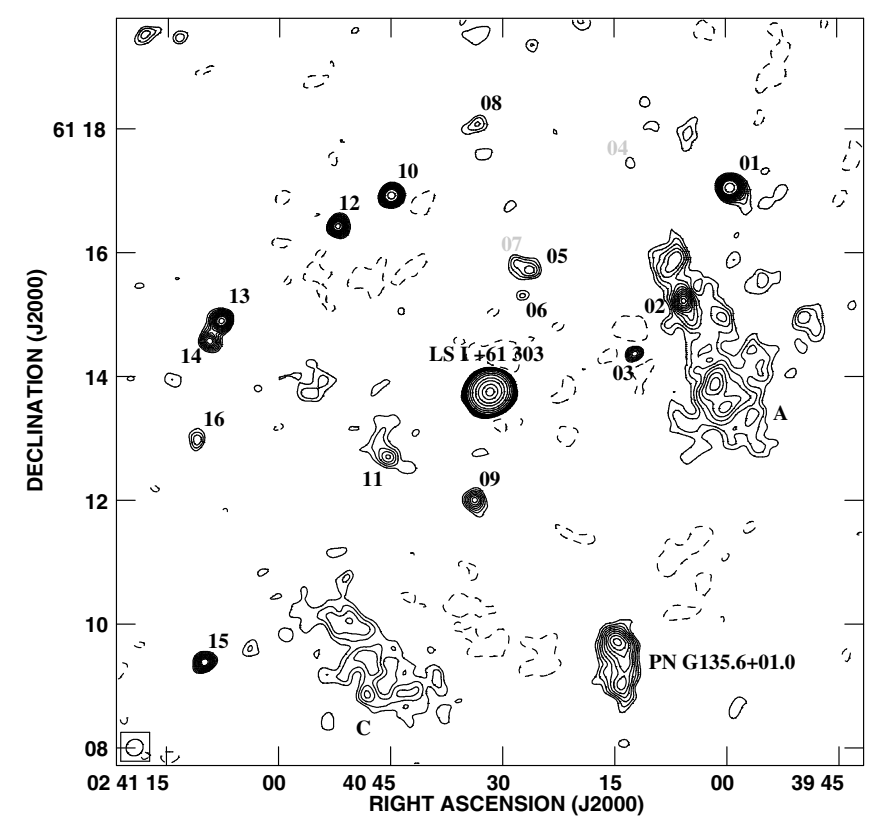

Fig. 1. Radio map of the LS I +61 303 environment at the $6 \mathrm{~cm}$ wavelength obtained after combining all VLA observations discussed in the text. The visibility data has been slightly tapered with a $15 \mathrm{k} \lambda$ Gaussian in order to enhance the extended emission in the field. The X-ray binary LS I +61 303 and the nearby planetary nebula G135.6+01.0 (Bond et al. 2003) are labelled and the other relevant sources numbered in right ascension order. Radio sources marked with grey numbers are better seen in the non-tapered map of Fig. 2. The rms noise is at the level of $14 \mu \mathrm{Jy}_{\text {beam }}{ }^{-1}$. The beam size is shown at the bottom left corner and corresponds to $17^{\prime \prime} .0 \times 15^{\prime \prime} .7$, with position angle of -53.3 . Contours shown are $-3,3,4,5,6,7,8,9,10,11,12,13,15,20,25,50,100,200$, $500,1000,2000$ and 3000 times the rms noise.

Hispano Alemán (CAHA) in Almería (Spain), one year before the Swift event. The OMEGA2000 camera was used and the images were taken through the $J, H$ and $K_{\mathrm{s}}$ filters. CAHA observations were processed following the standard procedures for sky background subtraction, flat-fielding and mediancombining of individual frames using the IRAF ${ }^{1}$ software package. Astrometry in the final frames was determined by identifying about twenty stars in the field for which positions were retrieved from the 2MASS catalog. The relevant part of the resulting image is shown in the right panel of Fig. 2. Photometric and astrometric data derived from this image are included in Table 2 for VLA radio sources with a near infrared counterpart. The errors in the photometric measurements are dominated by the uncertainty in the zero point, which is estimated to be about $0.1 \mathrm{mag}$ in all filters. The existence of a candidate counterpart was assessed based on the statistical parameter $r$ (Allington-Smith et al. 1982) defined to be:

$r=\sqrt{\frac{(\Delta \alpha \cos \delta)^{2}}{\sigma_{\alpha, \mathrm{IR}}^{2}+\sigma_{\alpha, \mathrm{rad}}^{2}}+\frac{(\Delta \delta)^{2}}{\sigma_{\delta, \mathrm{IR}}^{2}+\sigma_{\delta, \mathrm{rad}}^{2}}}$

where $\Delta \alpha$ and $\Delta \delta$ are the differences between the measured near infrared and radio positions, and $\sigma_{\alpha, \mathrm{IR}}, \sigma_{\alpha, \mathrm{rad}}, \sigma_{\delta \text {,IR }}$ and $\sigma_{\delta \text {,rad }}$ are the infrared and radio uncertainties in right ascension and declination.

The offsets between radio and near infrared positions are also listed in Table 2. A value of $r \leq 3$ is taken to be indicative of

1 http://iraf.noao.edu/ 

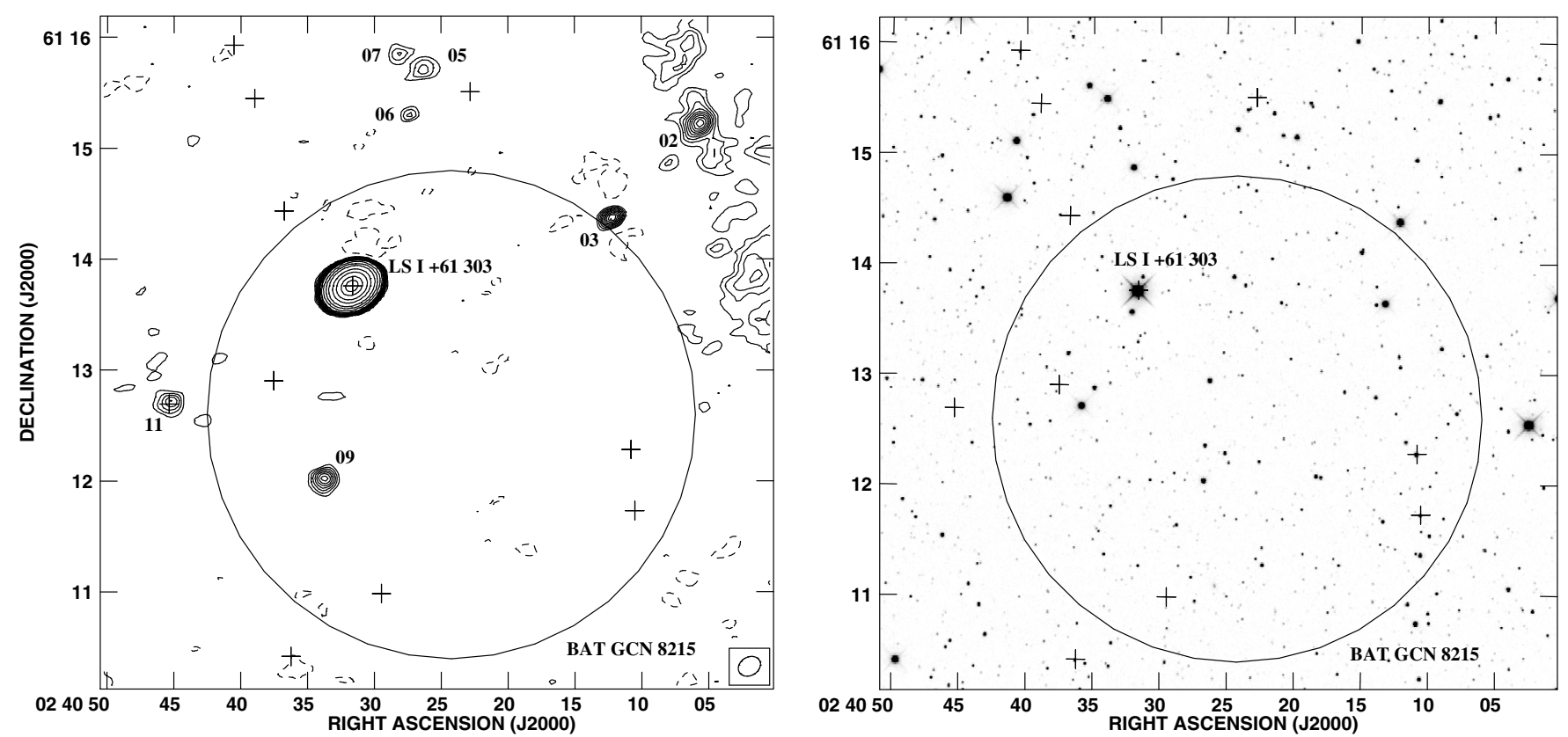

Fig. 2. Left. Zoomed contour radio map of the LS I +61 303 environment at $6 \mathrm{~cm}$ after combining all the VLA data used in this paper. Natural weight with no taper has been used. The big circle corresponds to the refined $90 \%$ confidence BAT location for the magnetar-like flare in the direction of LS I +61303 . The rms noise is at the level of $13 \mu \mathrm{Jy}$ beam ${ }^{-1}$. The beam size is shown at the bottom right corner and corresponds to $12^{\prime \prime} 7 \times 9$ '. $^{\prime}$, with position angle of -58.8 . Contours shown are $-3,3,4,5,6,7,8,9,10,11,12,13,15,20,25,50,100,200,500,1000,2000$ and 3000 times the rms noise. Crosses show the X-ray Chandra ACIS-I sources with a signal-to-noise ratio above 3. Right. The same field as observed in the near infrared $K_{\mathrm{s}}$-band with the CAHA $3.5 \mathrm{~m}$ telescope and the OMEGA2000 camera.

astrometric coincidence within the combined radio and near infrared position errors.

\section{X-ray sources within the Swift-BAT error circle}

We also used X-ray data from an observation of the LS I +61 303 field obtained with Chandra about two years before the Swift event using the standard ACIS-I setup in VF mode during a total of $49.9 \mathrm{ks}$. The data were reduced using the Chandra Interactive Analysis of Observations software package (CIAO v4.0) and CALDB v3.4.5. The analysis of LS I +61 303 data itself was discussed and its results reported in detail by Paredes et al. (2007). We used the CIAO tool wavdetect to obtain a list of candidate sources with data of a signal-to-noise ratio above $3 \sigma$. Most of the sources were very faint and the counts insufficient for a robust spectral analysis, so we obtained an exposure map of the observation and derived the photon fluxes and hardness ratios of the detected sources. In Table 3 , we present the fluxes in the $0.5-7.0 \mathrm{keV}$ band and the hardness ratio, defined as $(H-S) /(H+S)$, where $H$ is the flux in the $2.0-7.0 \mathrm{keV}$ band and $S$ is the flux in the $0.5-2.0 \mathrm{keV}$ band.

The X-ray observation completely covers the area observed in radio with the VLA. In this region, we found a total of 40 sources, excluding LS I +61 303 itself, 39 of which were previously unknown.

\section{Discussion and conclusions}

Our deep radio maps in Figs. 1 and 2 exhibit 16 compact radio sources in addition to LS I +61 303 with a peak flux density of a factor of four above the rms noise. When we restricted ourselves to the Swift-BAT error circle, three compact radio sources (labelled as \# 03, 09 and 11 in Table 2) appear to be located within or close to the refined BAT location. We measured their flux densities at $6 \mathrm{~cm}$ at the three epochs quoted in Table 1 separately, and no evidence of variability was found in any source. The VLA sources \# 03 and 09 are consistent with point-like near infrared counterparts (see Table 2). Source \# 03 coincides with a particularly bright object $\left(K_{\mathrm{s}}=10.0\right)$ and its observed colours (e.g. $J-K_{\mathrm{s}} \simeq 1.4$ ) are reminiscent of a giant star with a late spectral type, provided that interstellar extinction is not too high. Source \# 09 appears to be a highly reddened object with $J-K_{\mathrm{s}} \simeq 2.5 \mathrm{mag}$ and its stellar or extragalactic nature cannot be established based on our photometry alone. Neither of them is detected in our Chandra observations. On the other hand and using the same Eq. \# (1) criterion, VLA source \# 11 is coincident with a Chandra X-ray source (labelled as \# 27 in Table 3) but no infrared counterpart is detected. If either the magnetar-like burst behaviour or the X-ray activity presenting QPOs are unrelated to LS I +61 303, then these radio sources appear to be potential alternative counterparts for these detections.

We note that VLA source \# 11 is located at the centre of the extended radio source $\mathrm{D}\left(\alpha=02^{\mathrm{h}} 40^{\mathrm{m}} 45^{\mathrm{s}}, \delta=+61^{\circ} 12^{\prime} \cdot 7\right)$ reported by M98 which was proposed to be a possible large-scale lobe powered by LS I +61 303. In our new map (Fig. 1), this object resembles more closely a background radio galaxy with bent bipolar jets. The X-ray detection might then originate in the central core of the radio galaxy. In this interpretation, the connection of this source to a magnetar-like flare/QPOs appears unlikely since this kind of behaviour is not typical of an extragalactic object.

The new VLA maps confirm all the extended radio emission features reported by M98. A possible supernova remnant origin was tentatively considered by these authors but the low surface brightness and absence of extended X-rays rendered it unlikely. Revisiting this scenario seems timely because the magnetar-like emitter, even if it is not LS I +61 303, is naturally expected to be a galactic compact object created during a past supernova event. 
Table 2. Compact radio sources in the field around LS I +61 303 and their candidate near infrared counterparts.

\begin{tabular}{|c|c|c|c|c|c|c|c|c|c|c|}
\hline $\begin{array}{l}\text { VLA } \\
\text { Id. \# }\end{array}$ & $\begin{array}{l}\text { Right Ascension } \\
\text { (hms) }\end{array}$ & $\begin{array}{c}\text { Declination } \\
\left({ }^{\circ}{ }^{\prime \prime \prime}\right)\end{array}$ & $\begin{array}{l}\text { Peak flux dens. } \\
\left(\mathrm{mJy} \text { beam }^{-1}\right)\end{array}$ & $\begin{array}{l}\text { Total flux dens. } \\
\text { (mJy) }\end{array}$ & $\begin{array}{c}\Delta \alpha \cos \delta \\
\left({ }^{\prime \prime}\right)\end{array}$ & $\begin{array}{l}\Delta \delta \\
\left({ }^{\prime \prime}\right)\end{array}$ & $r$ & $\begin{array}{c}J \\
(\mathrm{mag})\end{array}$ & $\begin{array}{c}H \\
(\mathrm{mag})\end{array}$ & $\begin{array}{c}K_{\mathrm{s}} \\
(\mathrm{mag})\end{array}$ \\
\hline $01 *(a)$ & $023959.44(0.04)$ & $+611703.3(0.2)$ & $0.87(0.04)$ & $1.00(0.07)$ & +0.43 & +0.00 & 0.71 & 20.1 & 18.8 & 18.1 \\
\hline 02 & $024005.63(0.13)$ & $+611513.5(1.0)$ & $0.23(0.04)$ & $0.41(0.09)$ & - & - & - & - & - & - \\
\hline $03 *$ & $024012.22(0.11)$ & $+611422.4(0.7)$ & $0.20(0.04)$ & $0.15(0.05)$ & -1.01 & +0.70 & 1.17 & 11.4 & 10.3 & 10.0 \\
\hline 04 & $024012.77(0.24)$ & +61 $1725.9(1.4)$ & $0.11(0.04)$ & $0.10(0.06)$ & -0.94 & -0.10 & 0.27 & - & 18.8 & 17.7 \\
\hline 05 & $024026.32(0.47)$ & $+611543.2(2.4)$ & $0.08(0.04)$ & $0.17(0.10)$ & +0.50 & -0.90 & 0.38 & 18.0 & 17.1 & 16.0 \\
\hline 06 & $024027.34(0.34)$ & +61 $1518.6(1.9)$ & $0.07(0.04)$ & $0.06(0.06)$ & +0.43 & -0.10 & 0.10 & - & - & 18.2 \\
\hline 07 & $024028.13(0.43)$ & $+611550.8(2.6)$ & $0.07(0.04)$ & $0.12(0.09)$ & +4.47 & +3.80 & 1.62 & 15.0 & 14.5 & 14.3 \\
\hline 08 & $024033.33(0.26)$ & +61 $1805.8(1.9)$ & $0.12(0.04)$ & $0.24(0.10)$ & - & - & - & - & - & - \\
\hline $09 *$ & $024033.74(0.20)$ & +61 $1200.8(1.4)$ & $0.14(0.04)$ & $0.17(0.07)$ & -0.36 & -0.70 & 0.51 & 19.2 & 17.8 & 16.7 \\
\hline $10 *(a, b)$ & $024044.94(0.05)$ & $+611655.8(0.3)$ & $0.54(0.04)$ & $0.49(0.06)$ & +0.07 & +0.20 & 0.65 & 6.4 & 6.2 & 6.2 \\
\hline $11^{(a)}$ & $024045.32(0.33)$ & $+611242.3(2.0)$ & $0.10(0.04)$ & $0.17(0.09)$ & - & - & - & - & - & - \\
\hline $12 *$ & $024052.15(0.06)$ & +61 $1626.1(0.4)$ & $0.39(0.04)$ & $0.35(0.06)$ & +0.43 & +0.10 & 0.54 & - & - & 18.1 \\
\hline $13 *(\mathrm{a})$ & $024107.76(0.06)$ & $+611454.2(0.4)$ & $0.46(0.04)$ & $0.49(0.07)$ & +0.79 & +0.30 & 1.14 & 20.5 & 19.6 & 18.2 \\
\hline $14 *$ & $024109.36(0.07)$ & $+611434.5(0.5)$ & $0.34(0.04)$ & $0.34(0.07)$ & - & - & - & - & - & - \\
\hline $15^{*}$ & $024109.94(0.03)$ & +610923.0(0.2) & $1.16(0.04)$ & $1.00(0.06)$ & - & - & - & - & - & - \\
\hline $16^{*}$ & $024110.97(0.14)$ & $+611258.1(1.2)$ & $0.18(0.04)$ & $0.23(0.08)$ & - & - & - & - & - & - \\
\hline
\end{tabular}

* Sources already detected by M98.

(a) Candidate X-ray counterpart found for this source.

(b) Near infrared magnitudes taken from the 2MASS All-Sky Catalog of point sources.

Table 3. X-ray sources with a signal-to-noise ratio above $3 \sigma$ in the LS I +61 303 vicinity.

\begin{tabular}{|c|c|c|c|c|}
\hline $\begin{array}{l}\text { Chandra } \\
\text { Id. \# }\end{array}$ & $\begin{array}{c}\text { Right Ascension } \\
(\mathrm{hms})\end{array}$ & $\begin{array}{c}\text { Declination } \\
\left({ }^{\circ}, \prime \prime\right)\end{array}$ & $\begin{array}{c}\text { Flux } \\
\left(10^{-6} \mathrm{ph} \mathrm{cm}^{-2} \mathrm{~s}^{-1}\right)\end{array}$ & $\begin{array}{c}\text { Hardness } \\
\text { Ratio }\end{array}$ \\
\hline 01 & $023954.899(0.024)$ & $+611239.80(0.24)$ & $0.66(0.27)$ & $0.82(0.08)$ \\
\hline 02 & $023958.962(0.019)$ & +61 15 19.46(0.13) & $2.44(0.43)$ & $-0.93(0.35)$ \\
\hline 03 & $023959.445(0.016)$ & +61 $1703.53(0.10)$ & $3.98(0.53)$ & $0.33(0.09)$ \\
\hline 04 & $024001.019(0.024)$ & $+611645.73(0.14)$ & $2.43(0.43)$ & $-0.49(0.28)$ \\
\hline 05 & $024002.592(0.023)$ & $+611728.46(0.16)$ & $2.69(0.48)$ & $0.21(0.15)$ \\
\hline 06 & $024010.328(0.036)$ & $+611711.64(0.10)$ & $1.09(0.31)$ & $-0.48(0.46)$ \\
\hline 07 & $024010.521(0.017)$ & +611144.04(0.13) & $0.77(0.27)$ & $-0.86(0.70)$ \\
\hline 08 & $024010.804(0.014)$ & +61 $1216.98(0.13)$ & $1.01(0.29)$ & $-0.87(0.57)$ \\
\hline 09 & $024016.604(0.018)$ & +61 $1737.79(0.18)$ & $1.22(0.34)$ & $-0.44(0.43)$ \\
\hline 10 & $024022.166(0.030)$ & +61 $1757.30(0.16)$ & $1.09(0.33)$ & $-0.27(0.42)$ \\
\hline 11 & $024022.802(0.018)$ & $+610847.61(0.26)$ & $0.83(0.29)$ & $-1.13(0.80)$ \\
\hline 12 & $024022.821(0.007)$ & $+611530.59(0.05)$ & $2.42(0.40)$ & $0.02(0.17)$ \\
\hline 13 & $024024.507(0.015)$ & +61 $1720.90(0.13)$ & $1.75(0.36)$ & $0.59(0.09)$ \\
\hline 14 & $024026.729(0.011)$ & $+611620.20(0.13)$ & $1.37(0.33)$ & $-0.54(0.39)$ \\
\hline 15 & $024026.859(0.016)$ & $+611629.57(0.15)$ & $1.36(0.33)$ & $0.28(0.19)$ \\
\hline 16 & $024028.861(0.011)$ & +61 $1643.71(0.09)$ & $3.06(0.48)$ & $-0.44(0.24)$ \\
\hline 17 & $024029.471(0.011)$ & +611059.21(0.11) & $0.66(0.24)$ & $0.91(0.04)$ \\
\hline 18 & $024036.234(0.017)$ & +61 $1025.18(0.14)$ & $0.70(0.25)$ & $-0.66(0.65)$ \\
\hline 19 & $024036.754(0.015)$ & +61 14 26.11(0.14) & $0.77(0.30)$ & $0.44(0.24)$ \\
\hline 20 & $024037.526(0.009)$ & +61 $1254.33(0.19)$ & $0.53(0.22)$ & $-0.25(0.58)$ \\
\hline 21 & $024038.956(0.006)$ & $+611527.01(0.04)$ & $6.22(0.62)$ & $0.24(0.08)$ \\
\hline 22 & $024040.505(0.028)$ & +61 $1555.76(0.14)$ & $0.48(0.25)$ & $-0.06(0.61)$ \\
\hline 23 & $024044.029(0.019)$ & +611654.28(0.13) & $1.82(0.41)$ & $-0.59(0.38)$ \\
\hline 24 & $024044.233(0.040)$ & +61 $1816.45(0.19)$ & $0.97(0.33)$ & $0.02(0.37)$ \\
\hline 25 & $024044.384(0.022)$ & +61 $1728.50(0.09)$ & $4.70(0.58)$ & $-0.45(0.18)$ \\
\hline 26 & $024044.944(0.004)$ & $+611656.10(0.02)$ & $36.61(1.51)$ & $-0.81(0.08)$ \\
\hline 27 & $024045.346(0.019)$ & $+611241.61(0.12)$ & $0.51(0.22)$ & $0.45(0.26)$ \\
\hline 28 & $024047.666(0.013)$ & $+611617.56(0.06)$ & $3.05(0.76)$ & $-0.24(0.33)$ \\
\hline 29 & $024051.254(0.007)$ & +61 $1428.08(0.05)$ & $4.21(0.52)$ & $-0.61(0.21)$ \\
\hline 30 & $024053.383(0.024)$ & +61 $1555.31(0.09)$ & $0.88(0.28)$ & $0.43(0.20)$ \\
\hline 31 & $024053.754(0.014)$ & +61 $1429.60(0.11)$ & $0.61(0.24)$ & $-0.11(0.48)$ \\
\hline 32 & $024059.824(0.032)$ & +6109 31.73(0.25) & $0.82(0.30)$ & $-0.63(0.63)$ \\
\hline 33 & $024100.953(0.018)$ & +61 $1114.55(0.16)$ & $2.56(0.51)$ & $-0.15(0.24)$ \\
\hline 34 & $024102.787(0.010)$ & +61 $1400.39(0.07)$ & $4.14(0.52)$ & $0.58(0.06)$ \\
\hline 35 & $024104.715(0.036)$ & +61 $1532.62(0.23)$ & $1.85(0.43)$ & $0.08(0.23)$ \\
\hline 36 & $024107.025(0.033)$ & +61 $1628.63(0.20)$ & $1.28(0.36)$ & $-0.11(0.33)$ \\
\hline 37 & $024107.835(0.021)$ & +61 $1454.69(0.14)$ & $3.98(0.55)$ & $0.24(0.11)$ \\
\hline 38 & $024108.254(0.027)$ & +610806.78(0.23) & $4.82(0.65)$ & $-0.86(0.26)$ \\
\hline 39 & 0241 10.223(0.019) & +61 $1608.34(0.13)$ & $5.51(0.63)$ & $0.55(0.05)$ \\
\hline 40 & $024114.310(0.043)$ & $+611630.62(0.21)$ & $1.29(0.36)$ & $0.58(0.12)$ \\
\hline
\end{tabular}


Thus, we attempted to estimate the spectral index of the two brightest extended radio features located west $\left(\alpha=02^{\mathrm{h}} 40^{\mathrm{m}} 01^{\mathrm{s}}\right.$, $\left.\delta=+61^{\circ} 13^{\prime} \cdot 9\right)$ and south $\left(\alpha=02^{\mathrm{h}} 40^{\mathrm{m}} 47^{\mathrm{s}}, \delta=+61^{\circ} 09^{\prime} .5\right)$ of LS I +61 303 in Fig. 1 (sources A and C in M98) by combining our $6 \mathrm{~cm}$ map with the $20 \mathrm{~cm}$ archive data also quoted in Table 1 . In this process, the visibility data was appropriately constrained to match angular resolutions in both data sets. The resulting spectral index map is not conclusive enough due to the poor quality of the $20 \mathrm{~cm}$ data. Only the brightest parts of the western radio feature have a spectral index error below 0.1 suggesting a possible non-thermal origin $\left(S_{v} \propto v^{-0.7}\right)$. However, Chandra observations reveal no X-ray counterpart, even for this western extended radio feature. Improved long wavelength radio and X-ray observations are required to constrain more accurately the possibility of a faint supernova remnant associated with LS I +61 303 or any other source in the field. Concerning compact sources, only one source labelled as \# 15 was detected at $20 \mathrm{~cm}$. Its spectral index $(-0.6 \pm 0.2)$ may suggest a nonthermal emission mechanism for this source.

In conclusion, we have reported a handful of X-ray and radio sources within or close to the improved Swift-BAT location of the magnetar-like and QPO events towards LS I +61 303. No object displays any peculiar signature that could reveal a possible connection with these unusual phenomena. Although we cannot exclude them being alternative counterpart candidates, the most likely possibility is that LS I +61 303 is actually behind the observed flaring event.

Acknowledgements. The authors acknowledge support by grants AYA200768034-C03-02 and AYA2007-68034-C03-01 from the Spanish government, and
FEDER funds. This has been also supported by Plan Andaluz de Investigación of Junta de Andalucía as research group FQM322. The NRAO is a facility of the NSF operated under cooperative agreement by Associated Universities, Inc. This paper is also based on observations collected at the Centro Astronómico Hispano Alemán (CAHA) at Calar Alto, operated jointly by the Max-Planck Institut für Astronomie and the Instituto de Astrofísica de Andalucía (CSIC). This research made use of the SIMBAD database, operated at the CDS, Strasbourg, France.

\section{References}

Albert, J., Aliu, E., Anderhub, H., et al. 2008, [arXiv: 0806. 1865]

Allington-Smith, J. R., Perryman, M. A. C., Longair, M. S., Gunn, J. E., \& Westphal, J. A. 1982, MNRAS, 201, 331

Barthelmy, S. D., et al. 2008, GCN, 8215

Bond, H. E., Pollacco, D. L., \& Webbink, R. F. 2003, AJ, 125, 260

Bosch-Ramon, V., Paredes, J. M., Romero, G. E., \& Ribó, M. 2006, A\&A, 459, L25

De Pasquale, M., et al., 2008, GCN, 8209

Dubus, G. 2006, A\&A, 456, 801

Dubus, G., \& Giebels, B. 2008, ATel, 1715

Gregory, P. C., \& Taylor, A. R. 1978, Nature, 272, 704

Gregory, P. C., Taylor, A. R., Crampton, D., et al. 1979, AJ, 84, 1030

Hutchings, J. B., \& Crampton, D. 1981, PASP, 93, 486

Martí, J., Peracaula, M., Paredes, J. M., Massi, M., \& Estalella, R. 1998, A\&A, 329, 951 (M98)

Muñoz-Arjonilla, A. J., et al. 2008, ATel, 1740

Paredes, J. M., Ribó, M., Bosch-Ramon, V., et al. 2007, ApJ, 664, L39

Ray, P. S., \& Hartman, J. M. 2008, ATel, 1730

Rea, N., \& Torres, D. F. 2008, ATel, 1731

Romero, G. E., Okazaki, A. T., Orellana, M., \& Owocki, S. P. 2007, A\&A, 474, 15

Taylor, A. R., \& Gregory, P. C. 1982, ApJ, 255, 210

Taylor, A. R., \& Gregory, P. C. 1984, ApJ, 283, 273 